\title{
New Ferrocenyl Phenol Thiophosphines
}

KatarynaBretosh $^{\mathrm{a}, \mathrm{b}}$, Sandrine Vincendeau ${ }^{\mathrm{a}, \mathrm{b}}$, Lucie Routaboul, ${ }^{\mathrm{a}, \mathrm{b}} \mathrm{Jean}$-Claude Daran ${ }^{\mathrm{a}, \mathrm{b}}$, ZoiaVoitenko $^{\mathrm{c}}$, Eric Manoury ${ }^{\mathrm{a}, \mathrm{b} *}$

${ }^{\mathrm{a}}$ Université Toulouse III - Paul Sabatier, 118 route de Narbonne 31062 Toulouse Cedex 9

${ }^{\mathrm{b}}$ Laboratoire de Chimie de Coordination du CNRS, 205 route de Narbonne,

31077 Toulouse cedex 4

${ }^{\mathrm{c}}$ Taras Shevchenko National University of Kyiv, 64/13 Volodymyrska st., Kyiv 01601, Ukraine

eric.manoury@lcc-toulouse.fr

Keywords:ferrocene, Friedel-Crafts reaction, phosphine, phenol.

Two new enantiomerically pure ferrocenylphenol-thiophosphine compounds have been efficiently synthesized by a one pot procedure from enantiomerically pure alcohol $(R)$ (diphenylthiophosphinoferrocenyl)methanol 1 by a fully regioselectiveFriedel-Crafts reaction on phenols after strong acid activation. A mechanistic proposal through O-alkylation followed by rearrangement of the formed oxonium is proposed. All compounds have been and fully characterized by NMR $\left({ }^{1} \mathrm{H},{ }^{31} \mathrm{P}\right.$ and $\left.{ }^{13} \mathrm{C}\right)$ and High Resolution Mass Spectroscopy. The molecular structure of one of these ferrocene derivatives has been determined by $\mathrm{X}$ ray diffraction analysis on monocrystal.

\section{Introduction}

During the last years, we developedvariousplanar chiral ferrocene ligands for homogenous catalysis [1]. These ligands bear one phosphino group and one other coordination site [2] like a sulfur atom [3], a phosphorus atom [4], a nitrogen atom [5], an Nheterocyclic carbene [6], an oxygen atom [7] ,.... These ligands have been used in various catalytic asymmetric reactions, like asymmetric allylic substitution, asymmetric alkoxy- carbonylation, asymmetric hydrogenation, asymmetric Suzuki-Miyaura coupling [2-9],... Most of these ligands have been synthesized from a common intermediate: the alcohol $\mathbf{1}$ (see Scheme 1) which can be efficiently synthesized as racemic mixture or in an enantiomerically pure form [2]. The first reaction is a nucleophilic substitution of the hydroxy group of alcohol 1by, first, activation by a strong acid (tetrafluoroboric acid) probably yielding a ferrocene-stabilized carbocation [10] after protonation and loss of a water molecule and 
then trapping of the carbocation by the nucleophile.

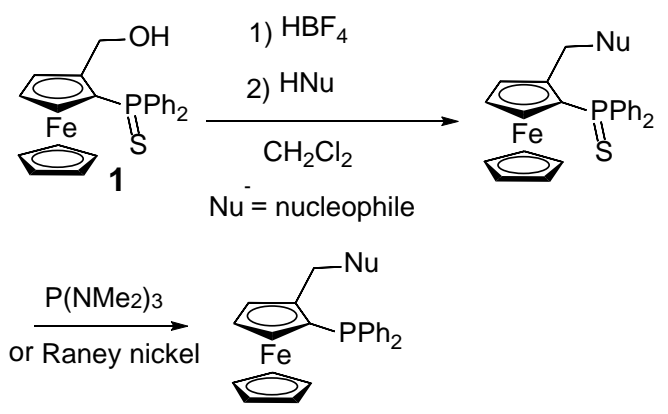

Scheme 1. Synthesis of planar chiral ferrocene ligands In this paper, we want to present our work on the use of phenols as nucleophiles in the reaction described above (sequential addition of $\mathrm{HBF}_{4}$ and a nucleophile on alcohol 1).

\section{Results and discussion}

As extension of our previous work on the development of phosphine bearing alkylether substituents [7b], we tried the reaction of enantiomerically pure $(\boldsymbol{R})-\mathbf{1}$ with phenol in order to obtain,after deprotection of the phosphine group,phosphine-arylether ligands (scheme 2).

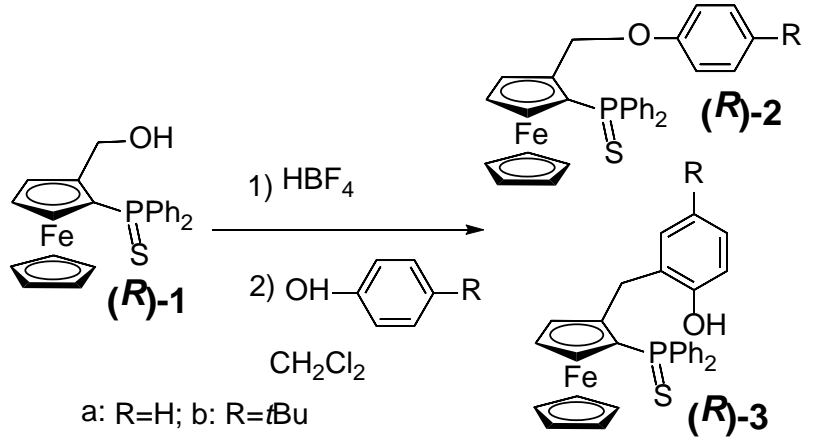

Scheme 2. Reaction of $\mathbf{1}$ with phenols.

However, no product of O-alkylation (R)-2a was observed after reaction but the only product was phenol (R)-3a from a Friedel-
Crafts reaction. Using phenols as substrates, Oalkylation and Friedel-Crafts reaction are wellknown to be competitive reactions with relative efficiencies as well as ortho/para ratios in Friedel-Crafts reaction which are highly sensitive to reaction conditions (solvent, temperature, catalyst,...).[11] The Friedel-Crafts reactivity is a new argument in favor of the involment of ferrocene-stabilized carbocation during the reaction of $(\boldsymbol{R})-\mathbf{1}$ with a strong acid.

As usually observed,Friedel-Crafts reaction on phenol should yield not only $(\boldsymbol{R})$-3a but also its regioisomer(R)-4a which it is not observed at all (see scheme 3). Very high selectivitiesfor the functionalization in the ortho positionof arenesare uncommon and are usually explained by a assistance of the neighboring substituents of the arenes.[12]In our case, we propose that this unusual regioselectivity is due to a directing effect of the hydroxyl group via a possible oxonium intermediate A obtained by reaction on the ferrocene-stabilized carbocation on the oxygen atom of the phenol, which rearranges into $(\boldsymbol{R})$-3a (see scheme 3).The involvement of such oxonium cations in Friedel-Crafts reaction has already been proposed. [11b] 

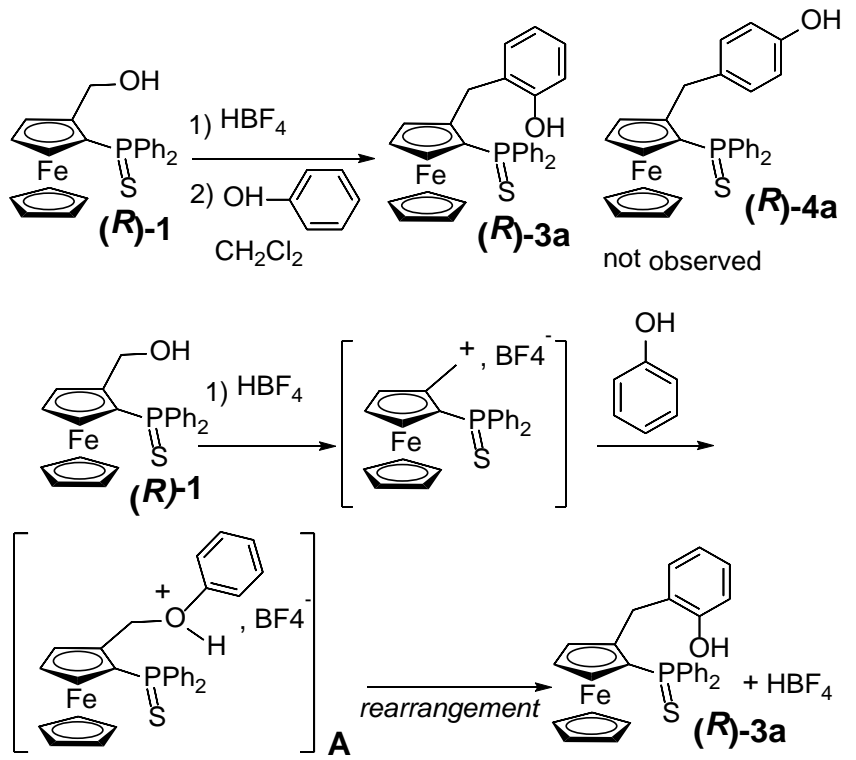

Scheme 3.Friedel-Crafts reaction of 1 with phenol.

The reaction was tried with another phenol, namely 4-tert-butyphenol. Once again, only one product (3b, scheme 2) was obtained in good yields from ortho-selective FriedelCrafts reaction.

Monocrystals suitable for X-ray diffraction analysis of compounds $(\boldsymbol{R})$-3acould be obtained by slow diffusion of hexane into a dichloromethane solution. Molecular view of the the structure of $(\boldsymbol{R})$-3ais represented in Figure 1.

The two Cp rings are nearly eclipsed with a twist angle $\tau$ of $4.5(2)^{\circ}$; they make a dihedral angle of $3.1(2)^{\circ}$. As observed in related ferrocenyl compounds the $\mathrm{S}$ atom is endo with respect to the $\mathrm{Cp}$ ring with a distance of 1.133(5) $\AA$ from the Cp ring. The phosphorus and the $\mathrm{CH}_{2}$ group are nearly in the $\mathrm{Cp}$ plane with distances from the plane being 0.092(4) $\AA$ and 0.066(4) $\AA$ respectively. There is a weak CH... intramolecular interaction between the
C212 carbon atom and the centroid of the C121—C126 phenyl ring (Table 2).

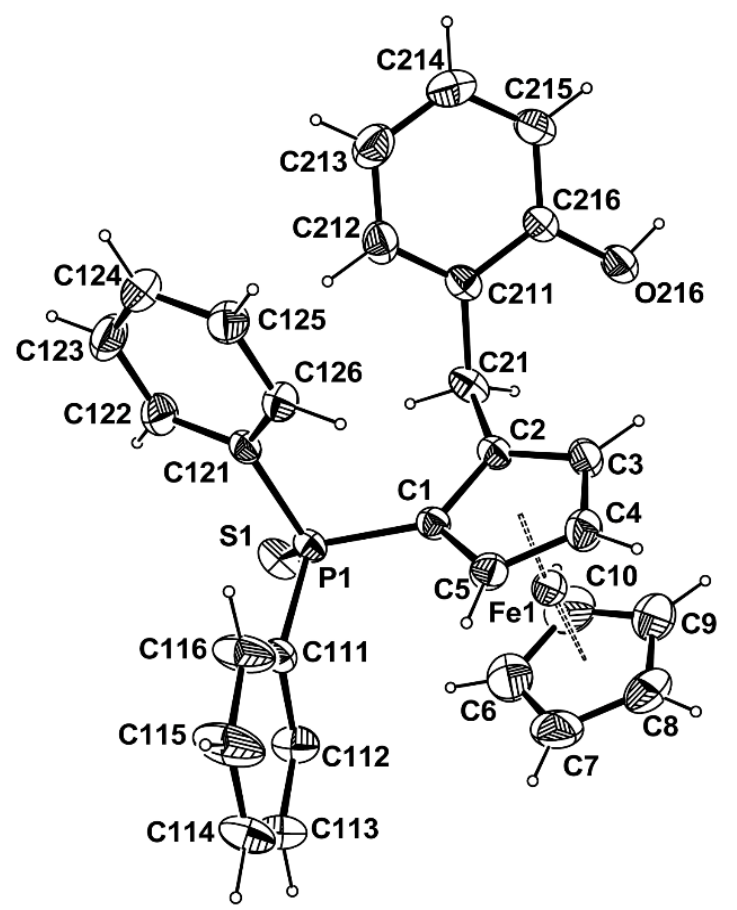

Figure 1: Molecular view of compound $(\boldsymbol{R})$-3a with the atom labeling scheme. Ellipsoids are drawn at the $50 \%$ probability level. $\mathrm{H}$ aroms are represented as small circle of arbitrary radii.

Table 1. Comparison of selected bond lengths $[\AA]$ and angles $\left[{ }^{\circ}\right]$ for $(\boldsymbol{R})$-3a.

\begin{tabular}{|ll|ll|}
\hline $\mathrm{P}(1)-\mathrm{S}(1)$ & $1.9665(8)$ & $\mathrm{P}(1)-\mathrm{C}(1)$ & $1.793(2)$ \\
\hline $\mathrm{P}(1)-\mathrm{C}(111)$ & $1.814(2)$ & $\mathrm{P}(1)-\mathrm{C}(121)$ & $1.810(2)$ \\
\hline $\mathrm{C}(1)-\mathrm{C}(5)$ & $1.435(3)$ & $\mathrm{C}(6)-\mathrm{C}(10)$ & $1.408(5)$ \\
\hline $\mathrm{C}(1)-\mathrm{C}(2)$ & $1.448(3)$ & $\mathrm{C}(6)-\mathrm{C}(7)$ & $1.414(5)$ \\
\hline $\mathrm{C}(2)-\mathrm{C}(3)$ & $1.422(3)$ & $\mathrm{C}(7)-\mathrm{C}(8)$ & $1.406(5)$ \\
\hline $\mathrm{C}(3)-\mathrm{C}(4)$ & $1.412(3)$ & $\mathrm{C}(8)-\mathrm{C}(9)$ & $1.403(5)$ \\
\hline $\mathrm{C}(4)-\mathrm{C}(5)$ & $1.407(3)$ & $\mathrm{C}(9)-\mathrm{C}(10)$ & $1.405(5)$ \\
\hline $\mathrm{C}(2)-\mathrm{C}(21)$ & $1.509(3)$ & $\mathrm{C}(21)-\mathrm{C}(211)$ & $1.510(3)$ \\
\hline $\mathrm{C}(111)-\mathrm{C}(112)$ & $1.375(3)$ & $\mathrm{C}(121)-\mathrm{C}(122)$ & $1.387(3)$ \\
\hline $\mathrm{C}(111)-\mathrm{C}(116)$ & $1.378(4)$ & $\mathrm{C}(121)-\mathrm{C}(126)$ & $1.389(3)$ \\
\hline $\mathrm{C}(112)-\mathrm{C}(113)$ & $1.380(3)$ & $\mathrm{C}(122)-\mathrm{C}(123)$ & $1.384(4)$ \\
\hline $\mathrm{C}(113)-\mathrm{C}(114)$ & $1.357(4)$ & $\mathrm{C}(123)-\mathrm{C}(124)$ & $1.361(4)$ \\
\hline $\mathrm{C}(114)-\mathrm{C}(115)$ & $1.356(4)$ & $\mathrm{C}(124)-\mathrm{C}(125)$ & $1.372(4)$ \\
\hline $\mathrm{C}(115)-\mathrm{C}(116)$ & $1.390(4)$ & $\mathrm{C}(125)-\mathrm{C}(126)$ & $1.383(3)$ \\
\hline $\mathrm{C}(211)-\mathrm{C}(212)$ & $1.392(4)$ & $\mathrm{C}(213)-\mathrm{C}(214)$ & $1.373(4)$ \\
\hline $\mathrm{C}(211)-\mathrm{C}(216)$ & $1.394(3)$ & $\mathrm{C}(214)-\mathrm{C}(215)$ & $1.378(4)$ \\
\hline $\mathrm{C}(212)-\mathrm{C}(213)$ & $1.374(4)$ & $\mathrm{C}(215)-\mathrm{C}(216)$ & $1.387(3)$ \\
\hline $\mathrm{C}(216)-\mathrm{O}(216)$ & $1.364(3)$ & & \\
\hline
\end{tabular}

\begin{tabular}{|lr|lr|}
\hline $\mathrm{C}(1)-\mathrm{P}(1)-\mathrm{C}(111)$ & $104.28(10)$ & $\mathrm{C}(1)-\mathrm{P}(1)-\mathrm{C}(121)$ & $104.17(10)$ \\
\hline $\mathrm{C}(1)-\mathrm{P}(1)-\mathrm{S}(1)$ & $117.12(8)$ & $\mathrm{C}(121)-\mathrm{P}(1)-\mathrm{C}(111)$ & $105.60(10)$ \\
\hline $\mathrm{C}(111)-\mathrm{P}(1)-\mathrm{S}(1)$ & $111.50(8)$ & $\mathrm{C}(121)-\mathrm{P}(1)-\mathrm{S}(1)$ & $113.12(8)$ \\
\hline $\mathrm{C}(1)-\mathrm{C}(2)-\mathrm{C}(21)$ & $127.6(2)$ & $\mathrm{C}(3)-\mathrm{C}(2)-\mathrm{C}(21)$ & $125.5(2)$ \\
\hline $\mathrm{C}(2)-\mathrm{C}(1)-\mathrm{P}(1)$ & $128.11(17)$ & $\mathrm{C}(5)-\mathrm{C}(1)-\mathrm{P}(1)$ & $124.50(16)$ \\
\hline $\mathrm{C}(3)-\mathrm{C}(2)-\mathrm{C}(1)$ & $106.86(19)$ & $\mathrm{C}(10)-\mathrm{C}(6)-\mathrm{C}(7)$ & $107.8(3)$ \\
\hline $\mathrm{C}(5)-\mathrm{C}(1)-\mathrm{C}(2)$ & $107.24(19)$ & $\mathrm{C}(8)-\mathrm{C}(7)-\mathrm{C}(6)$ & $107.9(3)$ \\
\hline $\mathrm{C}(4)-\mathrm{C}(3)-\mathrm{C}(2)$ & $109.1(2)$ & $\mathrm{C}(9)-\mathrm{C}(8)-\mathrm{C}(7)$ & $108.0(3)$ \\
\hline $\mathrm{C}(5)-\mathrm{C}(4)-\mathrm{C}(3)$ & $108.4(2)$ & $\mathrm{C}(8)-\mathrm{C}(9)-\mathrm{C}(10)$ & $108.4(3)$ \\
\hline $\mathrm{C}(4)-\mathrm{C}(5)-\mathrm{C}(1)$ & $108.4(2)$ & $\mathrm{C}(9)-\mathrm{C}(10)-\mathrm{C}(6)$ & $107.9(4)$ \\
\hline $\mathrm{C}(112)-\mathrm{C}(111)-\mathrm{P}(1)$ & $119.95(18)$ & $\mathrm{C}(116)-\mathrm{C}(111)-\mathrm{P}(1)$ & $121.45(18)$ \\
\hline $\mathrm{C}(122)-\mathrm{C}(121)-\mathrm{P}(1)$ & $120.77(18)$ & $\mathrm{C}(126)-\mathrm{C}(121)-\mathrm{P}(1)$ & $120.25(17)$ \\
\hline $\mathrm{C}(112)-\mathrm{C}(111)-\mathrm{C}(116)$ & $118.5(2)$ & $\mathrm{C}(122)-\mathrm{C}(121)-\mathrm{C}(126)$ & $119.0(2)$ \\
\hline
\end{tabular}




\begin{tabular}{|lr|ll|}
\hline $\mathrm{C}(111)-\mathrm{C}(112)-\mathrm{C}(113)$ & $120.5(2)$ & $\mathrm{C}(123)-\mathrm{C}(122)-\mathrm{C}(121)$ & $119.5(2)$ \\
\hline $\mathrm{C}(114)-\mathrm{C}(113)-\mathrm{C}(112)$ & $120.6(3)$ & $\mathrm{C}(124)-\mathrm{C}(123)-\mathrm{C}(122)$ & $121.2(2)$ \\
\hline $\mathrm{C}(115)-\mathrm{C}(114)-\mathrm{C}(113)$ & $119.9(2)$ & $\mathrm{C}(123)-\mathrm{C}(124)-\mathrm{C}(125)$ & $120.0(2)$ \\
\hline $\mathrm{C}(114)-\mathrm{C}(115)-\mathrm{C}(116)$ & $120.2(3)$ & $\mathrm{C}(124)-\mathrm{C}(125)-\mathrm{C}(126)$ & $119.8(2)$ \\
\hline $\mathrm{C}(111)-\mathrm{C}(116)-\mathrm{C}(115)$ & $120.3(3)$ & $\mathrm{C}(125)-\mathrm{C}(126)-\mathrm{C}(121)$ & $120.6(2)$ \\
\hline $\mathrm{C}(2)-\mathrm{C}(21)-\mathrm{C}(211)$ & $113.87(18)$ & & \\
\hline $\mathrm{C}(212)-\mathrm{C}(211)-\mathrm{C}(21)$ & $121.6(2)$ & $\mathrm{C}(216)-\mathrm{C}(211)-\mathrm{C}(21)$ & $120.8(2)$ \\
\hline $\mathrm{C}(212)-\mathrm{C}(211)-\mathrm{C}(216)$ & $117.6(2)$ & $\mathrm{C}(213)-\mathrm{C}(214)-\mathrm{C}(215)$ & $120.6(3)$ \\
\hline $\mathrm{C}(213)-\mathrm{C}(212)-\mathrm{C}(211)$ & $122.2(2)$ & $\mathrm{C}(214)-\mathrm{C}(215)-\mathrm{C}(216)$ & $120.0(2)$ \\
\hline $\mathrm{C}(214)-\mathrm{C}(213)-\mathrm{C}(212)$ & $119.1(3)$ & $\mathrm{C}(215)-\mathrm{C}(216)-\mathrm{C}(211)$ & $120.5(2)$ \\
\hline $\mathrm{O}(216)-\mathrm{C}(216)-\mathrm{C}(211)$ & $117.1(2)$ & $\mathrm{O}(216)-\mathrm{C}(216)-\mathrm{C}(215)$ & $122.5(2)$ \\
\hline
\end{tabular}

The molecules are linked by O-H...S interactions (Table 2) building helical chains parallel to the a axis (Figure 2).

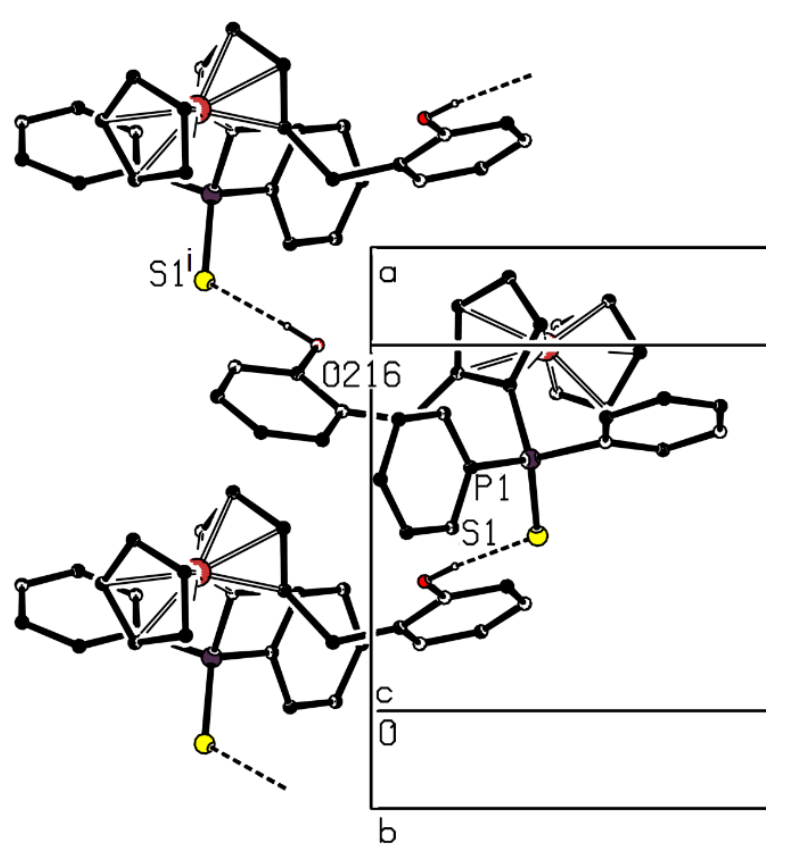
Figure 2: Partial packing view showing the helical chain ${ }_{13} \mathrm{C}\left\{{ }^{1} \mathrm{H}\right\}$ and ${ }^{31} \mathrm{P}\left\{{ }^{1} \mathrm{H}\right\}$ NMR spectra were
formed by the O-H...S hydrogen bond interaction: symmetry code (i): $x+1 / 2,-y+3 / 2,-z$.

Table 2. Hydrogen bonds data for (R)-3a.

\begin{tabular}{|c|c|c|c|}
\hline $\mathrm{D}-\mathrm{H} \ldots \mathrm{A}$ & $d(D-H)$ & $d(H \ldots A) d(D \ldots A)$ & $<(\mathrm{DHA})$ \\
\hline $\mathrm{O}(216)-\mathrm{H}(216) \ldots \mathrm{S}(1)^{\prime}$ & 0.82 & 2.423.2309(17) & 169.1 \\
\hline $\mathrm{C}(212)-\mathrm{H}(212) \ldots \mathrm{Cg}(1)$ & 0.93 & 3.645 & 145 \\
\hline
\end{tabular}

\section{Conclusions}

We discovered that the reaction of alcohol (R)-1with phenols after a strong acid activation yields only one phenol substituted in the ortho position with respect of the hydroxyl groupin good yields within 1 minute. We propose that this unusual regioselectivity in the Friedel-Crafts reaction is due to the formation of an oxonium intermediate as primary product of the reaction, which selectively decomposed into (R)-3. We now plan to evaluate the catalytic performances of the two enantiomericallypure thiophosphine-phenols (R)-3a and (R)-3b [13]and of their analogues with free phosphine groups instead of thiosphines.

\section{Experimental part}

General.All reactions were carried out under an argon atmosphere using standard Schlenk techniques. Solvents were carefully dried by conventional methods and distilled under argon before use. 2(diphenylthiophosphinoferrocenyl)methanol1[1 0]was synthesized by literature procedures. ${ }^{1} \mathrm{H}$, recorded with Bruker Avance 500 FT-NMR spectrometer. The resonances were calibrated relative to the residual solvent peaks and are reported with positive values downfield from TMS. For all characterized compounds, the peak assignments in the ${ }^{1} \mathrm{H}$ and ${ }^{13} \mathrm{C}$ NMR spectra were based on COSY, HSQC and HMBC 2D experiments. HRMS were obtained from dichloromethane solutions with a Xevo G2 
Q TOF spectrometer by the electrospray method.

Synthesis

thiodiphenylphosphino

hydroxyphenyl)methyl) ferrocene ( $R$ )-3a.

In a Schlenk tube,

(diphenylthiophosphinoferrocenyl)methanol(R)$\mathbf{1}(100 \mathrm{mg}, 0.23 \mathrm{mmol}$ ) was dissolved in $2 \mathrm{~mL}$ of dry dichloromethane. A $54 \%$ solution of tetrafluoroboric acid in ether $(100 \mu \mathrm{L}, 0.6757$ mmol) was then added. After 30 sec stirring, a solution of $400 \mathrm{mg}$ of phenol in $2 \mathrm{~mL}$ of dichloromethane (4.25mol, 18.5 eq.) was added by syringe. After $30 \mathrm{sec}$ of stirring, the crude materials were filtered on silica gel with ether as eluent. The crude product was then purified by flash chromatography on silicagel by using a pentane/ether (1/1, v/v) as eluent.After solvent evaporation in vacuum, 111mg of compound $(\boldsymbol{R})$-3aas a yellow solid.were obtained (yield = 95\%).

${ }^{1} \mathbf{H}$ NMR $\left(\delta\right.$ (ppm), $\left.\mathrm{CDCl}_{3}\right):$ 7.85-7.75 (2H, m, PPh 2$)$; 7.56-7.32 (6H, m, PPh 2 ); 7.24$7.18\left(2 \mathrm{H}, \mathrm{m}, \underline{\mathrm{Ph}}_{2}\right) ; 7.05$ (1H, m, $\left.\underline{\mathrm{PhOH}}\right) ; 6.94$ (1H, m, $\underline{\mathrm{PhOH}}) ; 6.73(1 \mathrm{H}, \mathrm{m}, \underline{\mathrm{PhOH}}) ; 6.62(1 \mathrm{H}$, m, $\underline{\mathrm{PhOH}}$ ); 4.58 (1H, m: substCp); 4.32 (1H, m: substCp); 4.28 (5H, s: Cp); 4.15 (1H, d, J = $15.2 \mathrm{~Hz}, \mathrm{AB}$ system, $\left.\mathrm{CH}_{2}\right) ; 3.84(1 \mathrm{H}, \mathrm{d}, \mathrm{J}=$ $15.2 \mathrm{~Hz}, \mathrm{AB}$ system, $\left.\mathrm{CH}_{2}\right) ; 3.67(1 \mathrm{H}, \mathrm{m}$ : substCp). ${ }^{13} \mathbf{C}$ NMR ( $\delta$ (ppm), $\left.\mathrm{CDCl}_{3}\right): 153.7$ (s: quat $\underline{\mathrm{ArOH}}$ ); 133.4 (d, J $\mathrm{PC}=86.3 \mathrm{~Hz}$ : quat $\mathrm{PPh}_{2}$ ); $132.4\left(\mathrm{~d}, \mathrm{~J}_{\mathrm{PC}}=86.9 \mathrm{~Hz}\right.$ : quat $\left.\mathrm{PPh}_{2}\right) ; 132.1$ (d,
$\left.\mathrm{J}_{\mathrm{PC}}=10.9 \mathrm{~Hz}: \mathrm{PPh}_{2}\right) ; 131.7\left(\mathrm{~d}, \mathrm{~J}_{\mathrm{PC}}=11.1 \mathrm{~Hz}\right.$ : $\left.\mathrm{PPh}_{2}\right) ; 131.5$ (d, JPC $\left.=3.1 \mathrm{~Hz}: \mathrm{PPh}_{2}\right) ; 131.2$ (d, $\left.\mathrm{J}_{\mathrm{PC}}=3.1 \mathrm{~Hz}: \mathrm{PPh}_{2}\right) ; 130.1$ (s: $\left.\underline{\mathrm{ArOH}}\right) ; 128.1$ (d,

(2- $\left.\mathrm{PPh}_{2}\right) ; 127.5$ (s: $\left.\underline{\operatorname{ArOH}}\right) ; 126.6$ (s: quat $\underline{\mathrm{ArOH}}$ ); 120.0 (s: $\underline{\operatorname{ArOH}}) ; 17.2$ (s: $\underline{\operatorname{ArOH}}) ; 92.7$ (d, JPC = $12.7 \mathrm{~Hz}$ : quatCp); 74.0 (d, $\mathrm{J}_{\mathrm{PC}}=13.0 \mathrm{~Hz}$ : substCp); 73.5 (d, J $\mathrm{J}_{\mathrm{PC}}=10.0 \mathrm{~Hz}$ : substCp); 73.0 $\left(\mathrm{d}, \mathrm{J}_{\mathrm{PC}}=95.7 \mathrm{~Hz}\right.$ : quatCp); 70.8 (s: Cp); 69.0 (d, $\mathrm{J}_{\mathrm{PC}}=10.6 \mathrm{~Hz}$ : substCp); $27.4\left(\mathrm{Fc}-\mathrm{CH}_{2}\right) .{ }^{31} \mathbf{P}$ NMR ( $\delta$ (ppm), $\left.\mathrm{CDCl}_{3}\right): 42.6 .[\alpha]_{\mathbf{D}}=+0.37$ $\left(\mathrm{CHCl}_{3}, \quad \mathrm{c}=0.5\right) . \mathbf{M S}(\mathbf{E S I}+)$ m/e: 509.0791 $(\mathrm{M}+1,100 \%)$ for $\mathrm{C}_{29} \mathrm{H}_{25} \mathrm{FeOPS}$.

\section{Synthesis of $\quad(R)-2-$}

thiodiphenylphosphino

((5-tert-butyl-2-

\section{hydroxyphenyl)methyl) ferrocene $(R)-3 b$.}

In a Schlenk tube, 2-thiodiphenyl phosphino (hydroxymethyl) ferrocene (R)-1 (100 g, $0.23 \mathrm{mmol}$ ) was dissolved in $2 \mathrm{~mL}$ of dry dichloromethane. A 54\% solution of tetrafluoroboric acid in ether $(100 \mu \mathrm{L}, 0.6757$ mmol) was then added. After $30 \mathrm{sec}$ stirring, a solution of $640 \mathrm{mg}$ of 4-tert-butyl-phenolin $2 \mathrm{~mL}$ of dichloromethane (4.25 mol, 18.5 eq.)was added by syringe.After $30 \mathrm{sec}$ of stirring, the crude materials were filtered on silica gel with ether as eluent. The crude product was then purified by flash chromatography on silicagel by using a pentane/ether (3/1, v/v) as eluent.95mg of compound (R)-3bas a yellow solid were obtained after evaporation (yield = 73\%). 
${ }^{* 1} \mathbf{H}$ NMR $\left(\delta(\mathrm{ppm}), \mathrm{CDCl}_{3}\right): 7.84-7.76$ $(2 \mathrm{H}, \mathrm{m}, \mathrm{PPh})$; 7.60-7.32 (6H, m, $\left.\underline{\mathrm{PPh}}_{2}\right) ; 7.25-$ $7.20\left(2 \mathrm{H}, \mathrm{m}, \mathrm{PPh}_{2}\right) ; 7.11(2 \mathrm{H}, \mathrm{d}, \mathrm{J}=2.5 \mathrm{~Hz}$, $\underline{\text { ArOH}) ; 6.98 ~(1 H, ~ d d, ~ J=2.5 ~ H z, ~ J=8.4 ~ H z, ~}$ $\underline{\operatorname{ArOH}}) ; 6.58(1 \mathrm{H}, \mathrm{d}, \mathrm{J}=8.4 \mathrm{~Hz}, \underline{\mathrm{ArOH}}) ; 4.57$ (1H, m: substCp); 4.30 (1H, m: substCp); 4.26 (5H, s: Cp); $4.06(1 \mathrm{H}, \mathrm{d}, \mathrm{J}=15.2 \mathrm{~Hz}, \mathrm{AB}$ system, $\left.\mathrm{CH}_{2}\right) ; 3.87(1 \mathrm{H}, \mathrm{d}, \mathrm{J}=15.2 \mathrm{~Hz}, \mathrm{AB}$ system, $\left.\mathrm{CH}_{2}\right)$; 3.67 (1H, m: substCp), $1.27(9 \mathrm{H}$, s, $\left.\mathrm{CH}_{3}\right) \cdot{ }^{13} \mathbf{C}$ NMR $\left(\delta(\mathrm{ppm}), \mathrm{CDCl}_{3}\right): 151.4$ (s: quat $\underline{\mathrm{ArOH}}$ ); 142.5 (s: quat $\underline{\mathrm{ArOH}}) ; 133.7$ (d, JP = 87.0 Hz: quat $\left.\mathrm{PPh}_{2}\right) ; 131.1\left(\mathrm{~d}, \mathrm{~J}_{\mathrm{PC}}=85 \mathrm{~Hz}\right.$ : quat $\left.\mathrm{PPh}_{2}\right) ; 132.1\left(\mathrm{~d}, \mathrm{~J}_{\mathrm{PC}}=10.7 \mathrm{~Hz}: \mathrm{PPh}_{2}\right)$; $131.7\left(\mathrm{~d}, \mathrm{~J}_{\mathrm{PC}}=10.7 \mathrm{~Hz}: \mathrm{PPh}_{2}\right) ; 131.5\left(\mathrm{~d}, \mathrm{~J}_{\mathrm{PC}}=\right.$ 3.0 Hz: $\mathrm{PPh}_{2}$ ); 131.1 (d, JPC $=3.0 \mathrm{~Hz}: \mathrm{PPh}_{2}$ ); $128.1\left(\mathrm{~d}, \mathrm{~J}_{\mathrm{PC}}=12.5 \mathrm{~Hz}: 2 \mathrm{C} \mathrm{PPh}_{2}\right.$ ); 127.0 (s: $\underline{\mathrm{ArOH}}) ; 125.9$ (s: quat $\underline{\mathrm{ArOH}}) ; 124.3$ (s: $\underline{\mathrm{ArOH}}) ;$ 116.5 (s: $\underline{\operatorname{ArOH}}) ; 93.0$ (d, J $\mathrm{J}_{\mathrm{PC}}=12.7 \mathrm{~Hz}$ : quatCp); 74.0 (d, $\mathrm{J}_{\mathrm{PC}}=13.1 \mathrm{~Hz}$ : substCp); 73.5 $\left(\mathrm{d}, \mathrm{J}_{\mathrm{PC}}=9.7 \mathrm{~Hz}:\right.$ substCp); $73.0\left(\mathrm{~d}, \mathrm{~J}_{\mathrm{PC}}=95.8\right.$ Hz: quatCp); 70.8 (s: Cp); 69.0 (d, $\mathrm{J}_{\mathrm{PC}}=10.5$ Hz: substCp); 45.8 (s: $\underline{\mathrm{C}}\left(\mathrm{CH}_{3}\right)$ ); 31.6 (s: $\left.\underline{\mathrm{CH}} 3\right)$; $27.7\left(\mathrm{Fc}-\mathrm{CH}_{2}\right),{ }^{31} \mathbf{P}$ NMR ( $\left.\delta(\mathrm{ppm}), \mathrm{CDCl}_{3}\right)$ : 42.6. $[\alpha]_{\mathbf{D}}=+0.20\left(\mathrm{CHCl}_{3}, \mathrm{c}=0.5\right) \cdot \mathbf{M S}(\mathbf{E S I}+)$ m/e: 563.1266 (M-1, 100\%), for $\mathrm{C}_{33} \mathrm{H}_{33} \mathrm{FeOPS}$.

\section{X-ray Structure Determination.}

Single crystal was mounted under inert perfluoropolyether at the tip of glass fibre and cooled in the cryostream of the Stoe IPDS diffractometer. Data were collected using the monochromatic MoK $\alpha$ radiation $(\lambda=0.71073)$.

The structures was solved by direct methods (SIR97)[14] and refined by least- squares procedures on F2 using SHELXL-97 [15]. All $\mathrm{H}$ atoms attached to carbon were introduced in calculation in idealised positions and treated as riding models. The drawing of the molecules was realised with the help of ORTEP32 [16]. Crystal data and refinement parameters are shown in Table 3.

Crystallographic data have been deposited with the Cambridge Crystallographic Data Centre as supplementary publication no. CCDC 1510434. Copies of the data can be obtained free of charge on application to the Director, CCDC, 12 Union Road, Cambridge CB2 1EZ, UK (fax: (+44) 1223-336-033; email: deposit@ccdc.cam.ac.uk).

Table 3. Crystal data and structurerefinement for $(\boldsymbol{R})$-3a .

\begin{tabular}{|c|c|}
\hline Identification code & (R)-3a \\
\hline Empirical formula & C29H25FeOPS \\
\hline Formula weight & 508.37 \\
\hline Temperature, $\mathrm{K}$ & $293(2)$ \\
\hline Wavelength, $\AA$ & $0.71073 \AA$ \\
\hline Crystal system & Orthorhombic \\
\hline Space group & Pbca \\
\hline $\mathrm{a}, \AA$ & 9.7020(9) \\
\hline $\mathrm{b}, \AA$ & $19.4446(12)$ \\
\hline $\mathrm{c}, \AA$ & $25.5137(19)$ \\
\hline$\alpha,{ }^{\circ}$ & 90.0 \\
\hline $\mathrm{b},{ }^{\circ}$ & 90.0 \\
\hline$\gamma,^{\circ}$ & 90.0 \\
\hline Volume, $\AA 3$ & $4813.2(6)$ \\
\hline $\mathrm{Z}$ & 8 \\
\hline Density (calc), Mg/m3 & 1.403 \\
\hline Abs. coefficient, mm-1 & 0.8000 \\
\hline $\mathrm{F}(000)$ & 2112 \\
\hline Crystal size, mm3 & $0.44 \times 0.44 \times 0.08$ \\
\hline 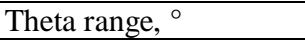 & 2.24 to 26.11 \\
\hline Reflections collected & 42493 \\
\hline Indpt reflections (Rint) & $4719(0.0648)$ \\
\hline Completeness, \% & 98.8 \\
\hline Absorption correction & Analytical \\
\hline $\begin{array}{l}\text { Max. / min. } \\
\text { transmission }\end{array}$ & $0.9371 / 0.6824$ \\
\hline Refinement method & $\mathrm{F} 2$ \\
\hline $\begin{array}{l}\text { Data } \\
\text { /restraints/parameters }\end{array}$ & 4719 / 0 / 299 \\
\hline Goodness-of-fit on F2 & 1.006 \\
\hline $\mathrm{R} 1, \mathrm{wR} 2[\mathrm{I}>2 \sigma(\mathrm{I})]$ & 0.0367, 0.0791 \\
\hline R1, wR2 (all data) & $0.0633,0.0881$ \\
\hline Residual density, e.Å-3 & $0.201 /-0.302$ \\
\hline
\end{tabular}




\section{Acknowledgements}

KB thanks the French Embassy in

Ukraine for grant funding.

\section{References}

[1] For recent reviews about chiral ferrocenyl ligands in asymmetric catalysis: (a) T. J. Colacot, Chem. Rev.2003, 103, 3101-3118.(b) R. C. J. Atkinson, V. C. Gibson, N. J. Long, Chem. Soc. Rev.2004, 33, 313-328.(c) R. Gomez Arrayas, J. Adrio, J. C. Carretero, Angew. Chem. Int. Ed.2006, 45, 7674-7715.(d) S. Toma, J. Csizmadiov6, М.Меиіаrovб, R. Sebesta, Dalton Trans.2014, 43, 1655716579.

[2] E. Manoury, R. Poli, "Phosphine-Containing Planar Chiral ferrocenes: Synthesis, Coordination Chemistry and Applications to Asymmetric Catalysis.” in the Series: Catalysis by Metal Complexes (CMCO), Volume 36 (Phosphorus Chemistry: Catalysis and Material Science Applications), edited by Maurizio Peruzzini and Luca Gonsalvi (Springer Verlag, Germany) 2011, 121-149.

[3] (a) L. Routaboul, S. Vincendeau, J.-C. Daran, E. Manoury, Tetrahedron: Asymmetry.2005, 16, 2685-2690. (b) L. Routaboul, S. Vincendeau, C.-O. Turrin, A.-M. Caminade, J.-P. Majoral, J.-C. Daran, E. Manoury, J. Organomet.Chem.,2007, 692, 1064-1073. (c) J. G.Lopez Cortes, O.Ramon, S. Vincendeau, D. Serra, F. Lamy, F., J.-C. Daran, E. Manoury, M. Gouygou, Eur. J. Inorg. ,Chem.,2006, 5148-5157. (d) M. Biosca, M. Coll, F. Lagarde, E. Brémond, L. Routaboul, E. Manoury, O. Pàmies, R. Poli, M. Diéguez, Tetrahedron,2016, 72, 2623-2631.

[4] J. G.Lopez Cortes, O.Ramon, S. Vincendeau, D. Serra, F. Lamy, F., J.-C. Daran, E. Manoury, M. Gouygou, Eur. J. Inorg. Chem.,2006, 5148-5157.

[5] (a) E. Manoury, J. S. Fossey, H. Aït-Haddou, J.-C. Daran, G.G. A. Balavoine, Organometallics,2000, 19, 3736-3739.(b) S. Mourgues, D. Serra, F. Lamy, S. Vincendeau, J.-C. Daran, E. Manoury, M. Gouygou, M, Eur. J. Inorg. Chem.,2003, 2820-2826. (c) A. Karpus, J.C. Daran, Z. Voitenko, E. Manoury, Fr. Ukr. J.Chem.,2015, 3, 131-139.

[6] (a) N. Debono, A. Labande, E. Manoury, Daran, R. Poli, Organometallics,2010, 29, 1879-1882. (b) P. Loxq,N. Debono, S. Gülcemal, J.-C. Daran, E. Manoury, R. Poli,B. Çetinkaya, A. Labande,New J. Chem.,2014, 38, 338-347.

[7](a) N. Mateus, L. Routaboul, S. Vincendeau, J.-C. Daran, E. Manoury, J. Organomet. Chem., 2006, 691, 2297-2310.(b) S. Bayda, A. Cassen, J.-C. Daran, C. Audin, R. Poli, E. Manoury, E. Deydier, J. Organomet. Chem., 2014, 772-773, 258-264.(c) A. Karpus, O. Yesypenko, V. Boiko, R. Poli, J.-C. Daran, Z. Voitenko, V. Kalchenko, E. Manoury, Eur. J. Org. Chem.,2016, 3386-3394.

[8] L. Diab, M. Gouygou, E. Manoury, P. KalckM. Urrutigoïty, TetrahedronLett.,2008, 49, 5186-5189.
[9] (a) E. LeRoux, R. Malacea, E. Manoury, R. Poli, L. Gonsalvi, M. Peruzzini, Adv. Synth. Catal.,2007,349, 309313. (b)E. M. Kozinets, G. A. Silantyev, N. V. Belkova, E. S. Shubina, R. Poli, E. Manoury, Russ.Chem. Bull.2013,750-756.

[10] (a) R. Gleiter, R. Seeger, H. Binder, E. Fluck, M. Cais, Angew. Chem. Int. Ed.,1972, 11, 1028-1029.(b) S. Allenmark, Tetrahedron Lett.,1974, 4, 371-374.(c) C. J. Richards, D. Hibbs, M. H. Hursthouse, Tetrahedron Lett.,1994, 35, 4215-4218.(d) S. Taudien, O. Riant, H. B. Kagan, Tetrahedron Lett.,1995, 36, 3513-3516.(e) A. Z. Kreidlin, F. M. Dolgushin, A. I. Yanovsky, Z. A. Kerzina, P. V. Petrovskii, J. Organomet. Chem.,2000, 616, 106-111.

[11] (a) G. A. Olah, D. Meidar, R. Malhotra, J. A. Olah, S. C. Narang, J.Catal., 1980, 61, 96-102. (b) M. Marczewski, J.-P. Bodibo, G. Perot, M. Guisnet, J.Mol.Catal.,1989, 50, 211-218. (c) G. Sarala Devi, D. Giridhar, B. M. Reddy, J.Mol.Catal. A: Chem.,2002, 181, 173-178. (d) K. Sreekumar, S. Sugunan, J.Mol.Catal. A: Chem.,2002, 185, 259-268. (e) A. S. Reddy, C. S. Gopinath, S. Chilukuri, J.Catal., 2006, 243, 278-291. (f) M. E. Sad, C. L. Padro, C. R. Apesteguia, Appl.Catal.A: Gen.,2008, 342, 40-48. (g) M. E. Sad, C. L. Padro, C. R. Apesteguia, Catalysis Today,2008, 133-135, 720-728.

[12] (a) J. P. Ryan, P. R. O’Connor, J.Am. Chem. Soc., 1952, 74, 5866-5869. (b) W. F. Burgoyne, Dale D. Dixon, Appl. Catal.,1990, 63, 117-127. (c) H. Suzuki, T. Takeuchi, T. Mori, J.Org. Chem.,1996, 61, 5944-5947. (d) T. Takebayashi, H. Hotta, A. Shono, S. Yoda, T. Furuya, K. Otake, Ind. Eng. Chem. Res..,2008, 47, 704-709. (e) N. I. Saper, B. B. Snider, J.Org. Chem.,2014, 79, 809-813.

[13]For selected exemples of the use of thiophosphines in catalysis, see reference [3a] and: (a) Y. K. Kim, T. Livinghouse, Y.Horino, J. Am. Chem. Soc.,2003, 125, 95609561. (b) X. Yu, T. J. Marks, Organometallics.2007, 26, 365376. (c) R. H. Platel, L. M. Hodgson, A. J. P. White, C. K. Williams, Organometallics.2007, 26, 4955-4963.(d)P. Hu, Z.J. Yao, J.-Q. Wang, G.-X. Jin, Organometallics,2011, 30, 4935-4940. (e) A. G. Jarvis, A. C. Whitwood, I.J. S. FairlambDalton Trans.,2011, 40, 3696-3702. (f) D. Ke, N. A. Espinosa, S. Mallet-Ladeira, J.Monot, B. Martin-Vaca, D.Bourissou, Adv. Synth. Catal.,2016, 358, 2324-2331. (g) J.Pan,Y. Wang, S. Chen, X; Zhang, Y. Wang, Z. Zhou,Tetrahedron,2016, 72, 240-246.

[14]G. M. Sheldrick, ActaCryst. A,2008, 64, 112-122.

[15]H. Flack, G. Bernadinelli, Chirality,2008,15, 681690.

[16]L. J. Farrugia, J. Appl. Cryst.,1997,30, 565. 